\title{
OPEN Dietary intake of tocopherols and risk of incident disabling dementia
}

\author{
Shoko Aoki ${ }^{1}$, Kazumasa Yamagishi ${ }^{1,2,3 凶}$, Koutatsu Maruyama ${ }^{4}$, Rie Kishida ${ }^{1}$, Ai Ikeda ${ }^{5}$, \\ Mitsumasa Umesawa ${ }^{1,6}$, Cui Renzhe ${ }^{7}$, Yasuhiko Kubota2, Mina Hayama-Terada ${ }^{2,8}$, \\ Yuji Shimizu ${ }^{2}$, Isao Muraki ${ }^{7}$, Hironori Imano ${ }^{7}$, Tomoko Sankai ${ }^{9}$, Takeo Okada ${ }^{2}$, \\ Akihiko Kitamura ${ }^{2,10}$, Masahiko Kiyama2 \& Hiroyasu Iso ${ }^{1,7}$
}

Tocopherols, strong antioxidants, may be useful in preventing dementia, but the epidemiological evidence is insufficient. We performed a community-based follow-up study of Japanese, the Circulatory Risk in Community Study, involving 3739 people aged 40-64 years at baseline (19851999). Incident disabling dementia was followed up from 1999 through 2020. For subtype analysis, we classified disabling dementia into that with and that without a history of stroke. Dietary intake of tocopherols (total, $\alpha, \beta, \gamma$, and $\delta$ ) were estimated using 24 -h recall surveys. During a median follow-up of 19.7 years, 670 cases of disabling dementia developed. Total tocopherol intake was inversely associated with risk of disabling dementia with multivariable hazard ratios ( $95 \%$ confidence intervals) of $0.79(0.63-1.00)$ for the highest versus lowest quartiles of total tocopherol intake ( $P$ for trend $=0.05$ ). However, the association was strengthened when further adjusted for $\alpha$-linolenic acid intake (Spearman correlation with total tocopherol intake $=0.93$ ), with multivariable hazard ratios of $0.50(0.34-0.74)$ ( $P$ for trend $=0.001$ ) but was weakened and nonsignificant when further adjusted for linoleic acid intake (Spearman correlation with total tocopherol intake $=0.92$ ), with multivariable hazard ratios of $0.69(0.47-1.01)$ ( $P$ for trend $=0.05$ ). Similar but nonsignificant inverse associations were observed for $\alpha-, \gamma^{-}$, and $\delta$-tocopherols but not for $\beta$-tocopherol. These results were similar regardless of the presence of a history of stroke. Dietary tocopherol intake was inversely associated with risk of disabling dementia, but its independent effect was uncertain owing to a high intercorrelation of $\alpha$-linolenic linoleic acids with total tocopherol intake. Even with such confounding, a diet high in tocopherols may help prevent the onset of dementia.

Vitamin E exists in four main forms, i.e., $\alpha-, \beta-, \gamma^{-}$, and $\delta$-tocopherols, and is abundant in edible plant fat and fish. It has been reported that vitamin E reduces reactive oxygen species ${ }^{1}$ and, in the human body, may prevent oxidized nucleic acids that could cause neurodegenerative diseases such as disabling dementia ${ }^{2}$. Therefore, tocopherols are expected to have clear antiatherosclerotic and neuronal protective functions that contribute to the prevention of such diseases. However, evidence from epidemiological studies in Europe and the United States has been controversial ${ }^{3-5}$, and no prospective studies have been reported in Asian populations.

We therefore investigated whether dietary tocopherol intake is associated with reduced risk of dementia in a community-based cohort study of Japanese men and women, whose mean intake of dietary tocopherols was lower than the intake reported to be adequate among men and women in Japanese communities ${ }^{6}$.

\footnotetext{
${ }^{1}$ Department of Public Health Medicine, Faculty of Medicine, and Health Services Research and Development Center, University of Tsukuba, Tennodai 1-1-1, Tsukuba 305-8575, Japan. ${ }^{2}$ Osaka Center for Cancer and Cardiovascular Disease Prevention, Osaka, Japan. ${ }^{3}$ baraki Western Medical Center, Chikusei, Japan. ${ }^{4}$ Department of Bioscience, Graduate School of Agriculture, Ehime University, Matsuyama, Japan. ${ }^{5}$ Department of Public Health, Juntendo University, Tokyo, Japan. ${ }^{6}$ Department of Public Health, Dokkyo Medical University, Mibu, Japan. ${ }^{7}$ Public Health, Department of Social Medicine, Osaka University Graduate School of Medicine, Suita, Japan. ${ }^{8}$ Yao City Public Health Center, Yao, Japan. ${ }^{9}$ Department of Public Health and Nursing, Faculty of Medicine, University of Tsukuba, Tsukuba, Japan. ${ }^{10}$ Research Team for Social Participation and Community Health, Tokyo Metropolitan Institute of Gerontology, Tokyo, Japan. ${ }^{\circledR}$ email: yamagishi.kazumas.ge@u.tsukuba.ac.jp
} 


\section{Participants and methods}

The Circulatory Risk in Communities Study (CIRCS) is an ongoing dynamic community-based prospective study involving five communities in Japan. Details of the CIRCS protocol have been described elsewhere ${ }^{7}$. In the present study, we included three communities, Ikawa (a rural community of Akita Prefecture in northeastern Japan), Kyowa (a rural community of Ibaraki Prefecture in mid-eastern Japan), and Minami-Takayasu (a suburb of Osaka Prefecture in mid-western Japan), where disabling dementia surveillance is being conducted.

We set the risk set as 3750 people aged 40-64 years living in these three communities who participated in an annual cardiovascular risk survey from 1985 to 1999 . They were followed up to confirm incident dementia from 1999 through 2020 (except for from April 2005 to April 2008, for which period the data were unavailable) in Kyowa; from 1999 to 2019 in Ikawa; and from 2006 to 2019 in Yao. The participants were primarily healthy at the time of the dietary surveys and we did not ask about a history of dementia in the surveys. Instead, we excluded persons who participated in the dietary survey at least 5 years before receiving a dementia diagnosis $(\mathrm{n}=2$ excluded) to minimize the possibility of reverse causation. We further excluded nine persons with invalid dietary data. In the end, 3739 participants were used for the analysis.

The intake of energy and nutrients from each meal was calculated on the basis of the Standard Tables of Food Composition in Japan, 2015 (7th revised edition) ${ }^{8}$. All nutrient variables were adjusted for energy intake using the nutrient residual model.

Diagnoses of disabling dementia were performed by attending physicians under the National Long-Term Care Insurance System, which is a compulsory insurance for all individuals aged 40 years or older in Japan . To apply for care under this system, the individual's attending physician is required to evaluate the individual's activities of daily living in relation to dementia. The criteria of disabling dementia were the same as those of our previous study (certified for disability under the long-term-care insurance program and the grade of activities of daily living related to dementia of $\geq \mathrm{IIa})^{10}$. The validation of these criteria was previously validated against diagnoses by certified psychiatrists with high specificity $(96 \%)$ and moderate sensitivity $(73 \%)^{11}$.

Potential risk factors for disabling dementia were measured at the baseline examination ${ }^{7}$. In brief, we measured the arterial systolic and fifth-phase diastolic blood pressures using standard mercury sphygmomanometers on the right arm of the participants, who were quietly seated after having rested for at least $5 \mathrm{~min}$. When the first systolic blood pressure reading was $\geq 140 \mathrm{mmHg}$ and/or the diastolic blood pressure was $\geq 90 \mathrm{mmHg}$, the blood pressure measurement was repeated. In this case, the second reading was used in the analysis; otherwise, was so the first reading. Height without shoes and weight in light clothing were measured, and body mass index was calculated as weight $(\mathrm{kg})$ divided by square of height $\left(\mathrm{m}^{2}\right)$. Face-to-face interviews were conducted to determine drinking (noncurrent or current) and smoking (never, ex, or current) statuses, and use of antihypertensive medication, cholesterol-lowering medication, and diabetes medication. Serum glucose and total cholesterol were measured at baseline without a fasting requirement. Diabetes mellitus was defined as fasting serum glucose $\geq 126 \mathrm{mg} / \mathrm{dL}$ or non-fasting serum glucose $\geq 200 \mathrm{mg} / \mathrm{dL}$ or being under medication for diabetes. In the present study, $90 \%$ of stroke occurrence was confirmed on the basis of computed tomography or magnetic resonance imaging using standardized criteria ${ }^{12}$, whilst the rest were determined using previously reported clinical criteria for diagnosis without imaging ${ }^{13,14}$. Stroke was defined as a rapid-onset focal neurological disorder persisting for $\geq 24$ h or until death.

The age- and sex-adjusted means and proportions of the characteristics of the study participants at the time of the dietary surveys were compared according to the quartiles of intakes of total, $\alpha-, \beta-, \gamma$-, and $\delta$-tocopherols by means of analyses of covariance. We conducted proportional hazards regression analysis using SAS 9.4 (SAS Institute, Cary, NC, USA). In model 1, we calculated age- and sex-adjusted and area-stratified hazard ratios and $95 \%$ confidence intervals for disabling dementia. We further adjusted for smoking status (never, former, current $1-20$ or $\geq 20$ cigarettes/day), intake of energy (continuous) and docosahexaenoic acid (DHA) + docosahexaenoic acid (EPA) ( $\omega-3$ polyunsaturated fatty acid of marine origin) in model 2 . Because of the high intercorrelations between total tocopherols and $\alpha$-linolenic acid (Pearson correlation coefficient $=0.93$ ), and linoleic acid (Pearson correlation coefficient $=0.92$ ), we further adjusted for $\alpha$-linolenic acid in model 3 and for linoleic acid in model 4 . To distinguish between different types of dementia, disabling dementia cases were classified into two categories: with a history of stroke and without a history of stroke, following the systematic stroke registration described elsewhere ${ }^{15}$ as well as self-reports in the dietary surveys. The end of the follow-up period was set for the end of 2015 for Kyowa and for the end of 2018 for Ikawa and Yao, considering the availability of the stroke registry data. All probability values for the statistical tests were 2 -tailed, and values below 0.05 were considered significant.

Informed consent was obtained from community leaders. Individual consent was not required for the analysis of this study, since it was conducted as a secondary use of data obtained for public health practice on cardiovascular disease prevention in the local community at that time. Adhering to relevant guidelines and regulations afterwards, participants were retrospectively given the opportunity to withdraw their data from analysis, and the consent was considered to have been obtained if the participant did not decline in this study.

This study was approved by the institutional review boards of both the Osaka Center for Cancer and Cardiovascular Disease Prevention and the University of Tsukuba.

Ethics approval. This study was approved by the institutional review boards of both the Osaka Center for Cancer and Cardiovascular Disease Prevention (approval number: R2-Rinri-4) and of the University of Tsukuba (approval number: 66-8).

Consent to participate. Informed consent was obtained from community leaders. Individual consent was not required for the analysis of this study, since it was conducted as a secondary use of data obtained for public health practice on cardiovascular disease prevention in the local community at that time. Adhering to relevant 


\begin{tabular}{|c|c|c|c|c|c|}
\hline & \multicolumn{5}{|c|}{ Total tocopherol } \\
\hline & Q1 (Low) & Q2 & Q3 & Q4 (High) & P for trend \\
\hline Median vitamin $\mathrm{E}^{\mathrm{a}}$ (mg/day) & 11.9 & 18.4 & 24.2 & 33.3 & \\
\hline Age (years) & 51.9 & 51.3 & 51.1 & 50.7 & $<0.0001$ \\
\hline Women (\%) & 39.2 & 51.3 & 61.9 & 71.0 & $<0.0001$ \\
\hline Body mass index $\left(\mathrm{kg} / \mathrm{m}^{2}\right)$ & 23.7 & 23.8 & 23.7 & 23.8 & 0.74 \\
\hline Current smoker (\%) & 37.7 & 28.7 & 23.4 & 19.5 & 0.28 \\
\hline Current drinker (\%) & 52.0 & 40.6 & 35.7 & 29.0 & 0.21 \\
\hline Systolic blood pressure ( $\mathrm{mmHg}$ ) & 132 & 131 & 131 & 129 & 0.07 \\
\hline Diastolic blood pressure $(\mathrm{mmHg})$ & 82.4 & 81.7 & 80.8 & 80.2 & 0.18 \\
\hline Antihypertensive medication (\%) & 13.0 & 11.1 & 12.8 & 12.6 & 0.51 \\
\hline Diabetes mellitus (\%) & 5.3 & 5.0 & 4.9 & 3.8 & 0.87 \\
\hline History of stroke (\%) & 1.2 & 1.0 & 0.7 & 1.2 & 0.61 \\
\hline Serum total cholesterol (mg/dL) & 199 & 201 & 199 & 202 & 0.21 \\
\hline Cholesterol-lowering medication (\%) & 1.7 & 2.1 & 2.5 & 2.4 & 0.73 \\
\hline Energy (kcal/day) & 2011 & 1955 & 1966 & 1973 & $<0.0001$ \\
\hline Carbohydrate (g/day) & 311 & 288 & 281 & 269 & $<0.0001$ \\
\hline Total fat (g/day) & 35.5 & 35.5 & 43.6 & 49.6 & $<0.0001$ \\
\hline Protein (g/day) & 67.4 & 70.2 & 72.2 & 71.8 & $<0.0001$ \\
\hline Total fiber (g/day) & 12.6 & 14.2 & 15.4 & 17.4 & $<0.0001$ \\
\hline Vegetables (g/day) & 227 & 258 & 296 & 322 & $<0.0001$ \\
\hline Fruit (g/day) & 132 & 142 & 144 & 160 & 0.33 \\
\hline Fish (g/day) & 98.9 & 100.1 & 98.8 & 88.5 & 0.08 \\
\hline Meats (g/day) & 46.7 & 49.5 & 50.4 & 48.0 & 0.12 \\
\hline Sodium (mg/day) & 4308 & 4528 & 4728 & 4774 & $<0.0001$ \\
\hline DHA + EPA (mg/day) & 991 & 979 & 968 & 806 & 0.03 \\
\hline a-Linolenic acid (mg/day) & 686 & 1139 & 1534 & 2354 & $<0.0001$ \\
\hline Linoleic acid (mg/day) & 4932 & 7390 & 9553 & 13,762 & $<0.0001$ \\
\hline
\end{tabular}

Table 1. Characteristics of participants aged 40-64 years, according to quartiles of total tocopherol intake at dietary surveys, CIRCS 1985-1999. 'anergy-adjusted values by nutrient residual model.

guidelines and regulations afterwards, participants were retrospectively given the opportunity to withdraw their data from analysis, and the consent was considered to have been obtained if the participant did not decline in this study.

Consent for publication. All the listed authors have approved the manuscript before submission.

\section{Results}

At baseline, the mean value of carbohydrate was inversely correlated, and the proportion of women and the mean values of total fat, total fiber, vegetables, sodium, a-linolenic acids, and linoleic acid were positively correlated with all of the tocopherol constituents (Table 1, Supplemental Tables 1-4).

As shown in Table 2, we observed an inverse association between dietary intakes of total tocopherol with risk of disabling dementia. The multivariable hazard ratios and $95 \%$ confidence intervals for persons with the second, third, and highest quartiles of total tocopherol in model 2 were 1.01 (0.82-1.25), 0.95 (0.77-1.18), and 0.79 (0.63-1.00), respectively, as compared with the lowest quartile ( $\mathrm{P}$ for trend $=0.05)$. Similar but nonsignificant associations with disabling dementia were observed for $\alpha^{-}, \gamma^{-}$, and $\delta$-tocopherols. No association was observed for $\beta$-tocopherol.

After further adjustment for linoleic acid, we still found an inverse association of dietary intake of total tocopherols with risk of incident dementia (model 4). The multivariable hazard ratios and 95\% confidence intervals for persons with the second, third, and highest quartiles of total tocopherol were $0.97(0.78-1.22)$, $0.88(0.67-1.16)$, and $0.69(0.47-1.01)$, respectively, as compared with the lowest quartiles (P for trend $=0.05)$. Adjustment for $\alpha$-linolenic acid strengthened these associations (model 3), whilst adjustment for linoleic acid attenuated the associations (model 4). It is noteworthy that the multivariable hazard ratios and $95 \%$ confidence intervals for the respective quartiles of $\alpha$-linolenic acid in model 2 were 1.05 (0.85-1.29), 0.93 (0.75-1.15), and $0.89(0.71-1.13)$, respectively, as compared with the lowest quartile $(P$ for trend $=0.21)$ and those for the respective quartiles of linoleic acid in model 2 were 0.95 (0.77-1.17), $0.86(0.69-1.07)$, and 0.90 (0.72-1.13), respectively, as compared with the lowest quartile $(\mathrm{P}$ for trend $=0.26)$ (data not shown).

These associations between dietary tocopherol intakes and disabling dementia were similarly observed when we analyzed the data for the participants with or without a history of stroke (Table 3). 


\begin{tabular}{|c|c|c|c|c|c|}
\hline & Q1 & Q2 & Q3 & Q4 & Trend $\mathbf{P}$ \\
\hline & \multicolumn{5}{|c|}{\begin{tabular}{|l|} 
Total tocopherol \\
\end{tabular}} \\
\hline Person-years & 14,363 & 14,974 & 14,980 & 15,365 & \\
\hline Number of cases & 178 & 179 & 168 & 145 & \\
\hline Model 1 & 1.00 & $0.99(0.81-1.22)$ & $0.93(0.75-1.16)$ & $0.79(0.63-1.00)$ & 0.04 \\
\hline Model 2 & 1.00 & $1.01(0.82-1.25)$ & $0.95(0.77-1.18)$ & $0.79(0.63-1.00)$ & 0.05 \\
\hline Model 3 & 1.00 & $0.89(0.71-1.12)$ & $0.74(0.56-0.98)$ & $0.50(0.34-0.74)$ & 0.001 \\
\hline \multirow[t]{2}{*}{ Model 4} & 1.00 & $0.97(0.78-1.22)$ & $0.88(0.67-1.16)$ & $0.69(0.47-1.01)$ & 0.05 \\
\hline & \multicolumn{5}{|c|}{ a-Tocopherol } \\
\hline Person-years & 14,480 & 15,011 & 15,023 & 15,167 & \\
\hline Number of cases & 177 & 148 & 175 & 170 & \\
\hline Model 1 & 1.00 & $0.73(0.59-0.91)$ & $0.89(0.72-1.10)$ & $0.79(0.64-0.99)$ & 0.16 \\
\hline Model 2 & 1.00 & $0.74(0.59-0.93)$ & $0.91(0.74-1.14)$ & $0.81(0.65-1.02)$ & 0.19 \\
\hline Model 3 & 1.00 & $0.74(0.59-0.92)$ & $0.90(0.72-1.13)$ & $0.80(0.62-1.03)$ & 0.25 \\
\hline \multirow[t]{2}{*}{ Model 4} & 1.00 & $0.75(0.60-0.94)$ & $0.94(0.66-1.18)$ & $0.85(0.66-1.09)$ & 0.53 \\
\hline & \multicolumn{5}{|c|}{$\beta$-Tocopherol } \\
\hline Person-years & 14,742 & 15,118 & 14,865 & 14,957 & \\
\hline Number of cases & 187 & 172 & 161 & 150 & \\
\hline Model 1 & 1.00 & $1.03(0.84-1.27)$ & $0.91(0.73-1.13)$ & $0.94(0.76-1.17)$ & 0.39 \\
\hline Model 2 & 1.00 & $1.04(0.84-1.28)$ & $0.92(0.74-1.15)$ & $0.93(0.74-1.16)$ & 0.48 \\
\hline Model 3 & 1.00 & $1.02(0.82-1.27)$ & $0.90(0.70-1.14)$ & $0.88(0.66-1.18)$ & 0.28 \\
\hline \multirow[t]{2}{*}{ Model 4} & 1.00 & $1.07(0.86-1.33)$ & $0.97(0.76-1.24)$ & $1.00(0.75-1.34)$ & 0.85 \\
\hline & \multicolumn{5}{|c|}{$\gamma$-Tocopherol } \\
\hline Person-years & 14,493 & 14,972 & 14,884 & 15,333 & \\
\hline Number of cases & 186 & 163 & 174 & 147 & \\
\hline Model 1 & 1.00 & $0.91(0.74-1.12)$ & $0.97(0.78-1.19)$ & $0.87(0.70-1.09)$ & 0.34 \\
\hline Model 2 & 1.00 & $0.92(0.74-1.14)$ & $0.97(0.79-1.20)$ & $0.86(0.68-1.08)$ & 0.39 \\
\hline Model 3 & 1.00 & $0.85(0.67-1.08)$ & $0.83(0.63-1.11)$ & $0.64(0.42-0.99)$ & 0.06 \\
\hline \multirow[t]{2}{*}{ Model 4} & 1.00 & $0.95(0.75-1.20)$ & $1.03(0.77-1.38)$ & $0.95(0.62-1.46)$ & 0.92 \\
\hline & \multicolumn{5}{|c|}{ 8-Tocopherol } \\
\hline Person-years & 14,572 & 14,740 & 15,210 & 15,160 & \\
\hline Number of cases & 175 & 161 & 169 & 165 & \\
\hline Model 1 & 1.00 & $0.93(0.75-1.15)$ & $0.90(0.73-1.11)$ & $0.86(0.69-1.07)$ & 0.16 \\
\hline Model 2 & 1.00 & $0.92(0.74-1.13)$ & $0.90(0.73-1.11)$ & $0.83(0.67-1.04)$ & 0.14 \\
\hline Model 3 & 1.00 & $0.88(0.70-1.10)$ & $0.83(0.66-1.06)$ & $0.73(0.55-0.99)$ & 0.04 \\
\hline Model 4 & 1.00 & $0.92(0.74-1.15)$ & $0.91(0.72-1.15)$ & $0.85(0.64-1.14)$ & 0.28 \\
\hline
\end{tabular}

Table 2. Multivariable hazard ratios and $95 \%$ confidence intervals of disability dementia according to quartiles of total tocopherol, $\alpha-, \beta-, \gamma$ - and $\delta$-tocopherol intakes. Model 1: Adjusted for age and sex, and stratified by area. Model 2: Multivariable model adjusted further for energy, smoking status, and DHA + EPA. Model 3: Multivariable model adjusted further for variables in model 2 and $\alpha$-linolenic acid. Model 4: Multivariable model adjusted further for variables in model 2 and linoleic acid.

Because of the lack of information regarding registration of dementia between 2005 and 2008 in Kyowa, we performed a sensitivity analysis starting from the follow-up from 2009. The associations did not change essentially (Supplemental Table 5).

\section{Discussion}

We found an inverse association between dietary tocopherol intake and risk of incident disabling dementia in the Japanese population. Since tocopherols, $\alpha$-linolenic acid, and linoleic acid are often found together in food sources (mainly vegetable oils), dietary intake of tocopherol was highly correlated with intakes of $\alpha$-linolenic acid and linoleic acid, and the observed tocopherol-dementia associations were changed after adjustment for $\alpha$-linolenic and linoleic acids.

Some animal and human studies support our findings that vitamin $\mathrm{E}$ is useful in preventing dementia. An antioxidant effect of $\alpha$-tocopherol was reported in a murine liver model where rats fed with supplemental vitamin $\mathrm{E}$ had higher liver $\alpha$-tocopherol levels and decreased lipid peroxidation after the introduction of an oxidizing agent ${ }^{1}$. Beyond antioxidant effects, $\gamma$-tocopherol increased sodium excretion in a dose-dependent manner in rats fed an $\mathrm{NaCl}$-rich $\operatorname{diet}^{16}$, suggesting that dietary $\gamma$-tocopherol may prevent vascular dementia associated with hypertension. Furthermore, triple transgenic mice for Alzheimer disease showed lower natural killer cell activity 


\begin{tabular}{|c|c|c|c|c|c|}
\hline & Q1 & Q2 & Q3 & Q4 & Trend P \\
\hline & \multicolumn{5}{|c|}{ Total tocopherol } \\
\hline Person-years & 14,363 & 14,974 & 14,980 & 15,365 & \\
\hline \multicolumn{6}{|c|}{ Dementia with a history of stroke } \\
\hline Number of cases & 41 & 33 & 21 & 18 & \\
\hline Model 1 & 1.00 & $0.89(0.56-1.40)$ & $0.62(0.36-1.06)$ & $0.60(0.34-1.06)$ & 0.04 \\
\hline Model 2 & 1.00 & $0.88(0.56-1.40)$ & $0.63(0.37-1.07)$ & $0.60(0.33-1.06)$ & 0.04 \\
\hline \multicolumn{6}{|c|}{ Dementia without a history of stroke } \\
\hline Number of cases & 109 & 109 & 111 & 88 & \\
\hline Model 1 & 1.00 & $0.95(0.73-1.24)$ & $0.96(0.73-1.26)$ & $0.77(0.57-1.03)$ & 0.09 \\
\hline \multirow[t]{2}{*}{ Model 2} & 1.00 & $0.97(0.74-1.27)$ & $0.98(0.74-1.28)$ & $0.77(0.57-1.03)$ & 0.09 \\
\hline & \multicolumn{5}{|c|}{ a-Tocopherol } \\
\hline Person-years & 14,480 & 15,011 & 15,023 & 15,167 & \\
\hline \multicolumn{6}{|c|}{ Dementia with a history of stroke } \\
\hline Number of cases & 30 & 33 & 28 & 22 & \\
\hline Model 1 & 1.00 & $1.09(0.66-1.80)$ & $1.03(0.61-1.74)$ & $0.84(0.47-1.48)$ & 0.52 \\
\hline Model 2 & 1.00 & $1.11(0.67-1.84)$ & $1.03(0.61-1.76)$ & $0.83(0.46-1.50)$ & 0.51 \\
\hline \multicolumn{6}{|c|}{ Dementia without a history of stroke } \\
\hline Number of cases & 112 & 83 & 110 & 112 & \\
\hline Model 1 & 1.00 & $0.60(0.45-0.80)$ & $0.80(0.61-1.05)$ & $0.73(0.55-0.96)$ & 0.15 \\
\hline \multirow[t]{2}{*}{ Model 2} & 1.00 & $0.61(0.45-0.81)$ & $0.82(0.63-1.08)$ & $0.75(0.57-1.00)$ & 0.22 \\
\hline & \multicolumn{5}{|c|}{$\boldsymbol{\beta}$-Tocopherol } \\
\hline Person-years & 14,742 & 15,118 & 14,865 & 14,957 & \\
\hline \multicolumn{6}{|c|}{ Dementia with a history of stroke } \\
\hline Number of cases & 41 & 28 & 24 & 20 & \\
\hline Model 1 & 1.00 & $0.82(0.51-1.34)$ & $0.77(0.46-1.29)$ & $0.72(0.42-1.25)$ & 0.24 \\
\hline Model 2 & 1.00 & $0.83(0.51-1.35)$ & $0.80(0.48-1.34)$ & $0.73(0.41-1.28)$ & 0.27 \\
\hline \multicolumn{6}{|c|}{ Dementia without a history of stroke } \\
\hline Number of cases & 116 & 106 & 103 & 92 & \\
\hline Model 1 & 1.00 & $1.05(0.80-1.36)$ & $0.92(0.70-1.20)$ & $0.93(0.70-1.23)$ & 0.45 \\
\hline \multirow[t]{2}{*}{ Model 2} & 1.00 & $1.05(0.80-1.37)$ & $0.93(0.70-1.22)$ & $0.91(0.68-1.21)$ & 0.37 \\
\hline & \multicolumn{5}{|c|}{$\gamma$-Tocopherol } \\
\hline Person-years & 14,493 & 14,972 & 14,884 & 15,333 & \\
\hline \multicolumn{6}{|c|}{ Dementia with a history of stroke } \\
\hline Number of cases & 41 & 24 & 30 & 18 & \\
\hline Model 1 & 1.00 & $0.68(0.41-1.13)$ & $0.94(0.58-1.52)$ & $0.67(0.38-1.19)$ & 0.34 \\
\hline Model 2 & 1.00 & $0.68(0.41-1.14)$ & $0.95(0.58-1.54)$ & $0.67(0.37-1.20)$ & 0.36 \\
\hline \multicolumn{6}{|c|}{ Dementia without a history of stroke } \\
\hline Number of cases & 116 & 107 & 107 & 87 & \\
\hline Model 1 & 1.00 & $0.94(0.72-1.23)$ & $0.95(0.73-1.25)$ & $0.85(0.64-1.14)$ & 0.31 \\
\hline \multirow[t]{2}{*}{ Model 2} & 1.00 & $0.95(0.72-1.24)$ & $0.95(0.72-1.25)$ & $0.83(0.62-1.12)$ & 0.25 \\
\hline & \multicolumn{5}{|c|}{$\delta$-Tocopherol } \\
\hline Person-years & 14,572 & 14,740 & 15,210 & 15,160 & \\
\hline \multicolumn{6}{|c|}{ Dementia with a history of stroke } \\
\hline Number of cases & 41 & 24 & 22 & 26 & \\
\hline Model 1 & 1.00 & $0.64(0.38-1.06)$ & $0.58(0.35-0.98)$ & $0.75(0.45-1.24)$ & 0.24 \\
\hline Model 2 & 1.00 & $0.63(0.38-1.04)$ & $0.57(0.34-0.97)$ & $0.74(0.44-1.22)$ & 0.23 \\
\hline \multicolumn{6}{|c|}{ Dementia without a history of stroke } \\
\hline Number of cases & 108 & 107 & 110 & 92 & \\
\hline Model 1 & 1.00 & $0.96(0.74-1.26)$ & $0.95(0.73-1.24)$ & $0.76(0.57-1.01)$ & 0.07 \\
\hline Model 2 & 1.00 & $0.95(0.72-1.24)$ & $0.94(0.72-1.23)$ & $0.73(0.55-0.98)$ & 0.04 \\
\hline
\end{tabular}

Table 3. Multivariable hazard ratios and $95 \%$ confidence intervals of disability dementia according to quartiles of total tocopherol, $\alpha-, \beta-, \gamma$ - and $\delta$-tocopherol intakes. Model 1: Adjusted for age and sex, and stratified by area. Model 2: Multivariable model adjusted further for energy, smoking status, and DHA + EPA. 
than that of the controls before the onset of Alzheimer disease ${ }^{17}$, whilst dietary supplementation of vitamin $\mathrm{E}$ is known to enhance natural killer cell proliferation in older adults ${ }^{18}$.

Dietary studies in humans also reported generally similar results. The Rotterdam Study of 5395 participants, aged 55 years or older and with a mean follow-up of 9.6 years, showed that participants in the highest tertile of dietary vitamin $\mathrm{E}$ had a lower risk of developing dementia than did those in the lowest tertile (HR, 0.76; 95\% CI $0.60-0.96)^{5}$. As for Alzheimer disease, a meta-analysis that enrolled 904 dementia patients and 1153 controls in Europe reported that Alzheimer disease patients had lower concentrations of serum vitamin $\mathrm{E}$ than did the healthy controls (weighted mean difference $=-6.811 \mu \mathrm{mol} / \mathrm{L}, 95 \% \mathrm{CI}-8.998$ to $-4.625, \mathrm{P}<0.001)^{19}$. Another prospective study of 815 US residents aged 65 years or older, free of dementia at baseline, and with a median follow-up of 3.9 years showed that dietary vitamin E intake was inversely associated with risk of Alzheimer disease only among persons who were $A P O E \varepsilon 4$ noncarriers. In that study, the multivariable relative risks and $95 \%$ confidence intervals for the second, third, fourth, and highest quartiles of vitamin E intake were $0.63(0.20-2.01)$, 0.34 (0.13-0.89), $0.23(0.08-0.67)$, and 0.17 (0.06-0.47), respectively, as compared with the lowest quartile ${ }^{3}$. Although we did not have APOE information for our participants, the reported $\varepsilon 4$ allele frequency in Japanese individuals $(11.6 \%)$ is not high when compared with that in white individuals $(24.0 \%)^{20}$, and thus, this $A P O E$ genotype is unlikely to have largely impacted our results. No prospective study has been conducted to show the association between dietary tocopherol intake and risk of vascular dementia.

In contrast, previous studies did not show any associations between dietary vitamin $\mathrm{E}$ and risk of dementia. A study of 980 participants aged 65 years or older with a mean follow-up of 4 years, in the Washington HeightsInwood Columbia Aging Project, showed weaker and nonsignificant associations between dietary vitamin $\mathrm{E}$ intake and decreased risk of Alzheimer disease $(\mathrm{P} \text { for trend }=0.83)^{4}$. The other studies reported that use of supplemental vitamin $\mathrm{E}$ was not associated with a decreased risk of Alzheimer disease $\mathrm{e}^{21,22}$. As for randomized controlled studies, a study of 3786 healthy men aged 60 years or older showed that vitamin E supplementation did not prevent dementia: the yielded hazard ratio was 0.88 (95\% CI 0.64-1.20) as compared with the placebo ${ }^{23}$. This discrepancy might be due to some factors that may affect the bioavailability of vitamin E; for example, it has been reported that age, sex, obesity, smoking, low-density lipoprotein cholesterol, high-density lipoprotein cholesterol, triglycerides, and fasting blood glucose affect serum vitamin E concentrations ${ }^{24}$. Differences in the follow-up periods may also have influenced each result.

The effect of $\omega-3$ polyunsaturated fatty acids and $\omega-6$ polyunsaturated fatty acids, which may be beneficial in the prevention of dementia, should be taken into account because tocopherols, $\omega-3$ polyunsaturated fatty acids, and $\omega-6$ polyunsaturated fatty acids, especially $\alpha$-linolenic acid and linoleic acid, are found in shared food sources. In the CIRCS, we previously reported that serum a-linolenic acid, but not EPA or DHA, was inversely associated with risk of disabling dementia ${ }^{25}$. In the present study, we observed that the association between intakes of all tocopherol constituents and risk of disabling dementia were strengthened when we adjusted for dietary $\alpha$-linolenic acid intake. Since edible plant fat and fish abundantly contain both tocopherol and $\alpha$-linolenic acid, the strong intercorrelation $(\mathrm{r}=0.93)$ dictates that the individual impacts of these nutrients cannot be completely distinguished in a real-world observational study. Similarly, studies reported that simultaneous intake of tocopherol, $a$-linolenic and linoleic acid from foods and dietary patterns were associated with lowered risk of dementia. A 3.9-year cohort study of 2148 persons aged 65 years or older showed that dietary patterns characterized by a diet rich in $\omega-3$ PUFA, $\omega-6$ PUFA, vitamin E, and folate were significantly associated with a lower risk of incident Alzheimer disease $(\mathrm{P} \text { for trend }=0.01)^{26}$.

The strength of the present study involved the use of a community-based sample of relatively young individuals (aged 59.7 years on average) and a long follow-up (24.1 years on average). Our findings are thus likely to be applicable to other Asian populations. Furthermore, we estimated intakes of $\alpha, \beta, \gamma$, and $\delta$-tocopherols and evaluated the associations between intake of each of these tocopherols and risk of disabling dementia.

Several limitations of this study should be noted. First, attending physicians diagnosed disabling dementia, but the criteria for such diagnoses were validated against diagnosis by certified psychiatrists in a previous study ${ }^{11}$. The sensitivity and specificity for these diagnoses were $73 \%$ and $96 \%$. Second, we analyzed dietary survey data using 24-h recall surveys, which may be insufficient to reflect typical diets for individuals; we previously performed a reproducibility study of 232 Japanese residents aged 40-69 years and reported that the correlation coefficients of total tocopherols examined a year apart in the same season throughout 1973-1999 was moderate ( 0.36 for both men and women $)^{27}$. In the present study period (1984-2000), the correlation coefficients between the tocopherol values examined a year apart were moderate to high except for those of $\gamma$-tocopherol $(r=0.44$ for total tocopherol, $\mathrm{r}=0.76$ for $\alpha$-tocopherol, $\mathrm{r}=0.72$ for $\beta$-tocopherol, $\mathrm{r}=0.25$ for $\gamma$-tocopherol, and $\mathrm{r}=0.80$ for $\delta$-tocopherol). Third, we did not have data on the type of dementia (i.e., Alzheimer or vascular dementia), but we did have information on dementia with or without a history of stroke. Although the number of cases was relatively small, the association of dietary tocopherols intake was similarly observed with disabling dementia regardless of the presence of a history of stroke (Table 3). Finally, since we did not survey prevalent dementia at baseline, we set the baseline at least 5 years before the beginning of the dementia survey (median follow-up $=19.7$ years) to reduce the possibility of reverse causation. In addition, we did not have the information on the dementia registry in Kyowa between 2005 and 2008. We performed sensitivity analyses following up all participants from 2009 and found no change in the results.

In conclusion, dietary tocopherol intake was inversely associated with risk of disabling dementia among Japanese, suggesting that persons with a diet consisting of sufficient tocopherol intake are less likely to develop disabling dementia. However, it is difficult to separate the effect of tocopherol intake from that of $a$-linolenic acid owing to the strong correlation with dietary tocopherol intake. Even with such confounding, a diet high in tocopherols may help prevent the onset of dementia. 


\section{Data availability}

The datasets generated during and/or analyzed during the current study are not publicly available owing to the restriction policy of the CIRCS committee but are available from the corresponding author upon reasonable request and with permission of the CIRCS committee.

\section{Code availability}

The code for the study analyses is available from the corresponding author upon reasonable request and with permission of the CIRCS committee.

Received: 12 November 2020; Accepted: 27 July 2021

Published online: 12 August 2021

\section{References}

1. Ham, A. J. \& Liebler, D. C. Antioxidant reactions of vitamin E in the perfused rat liver: Product distribution and effect of dietary vitamin E supplementation. Arch Biochem. Biophys. 339(1), 157-164 (1997).

2. Sheng, Z. et al. 8-Oxoguanine causes neurodegeneration during MUTYH-mediated DNA base excision repair. J. Clin. Invest. 122(12), 4344-4361 (2012).

3. Morris, M. C. et al. Dietary intake of antioxidant nutrients and the risk of incident Alzheimer disease in a biracial community study. JAMA 287(24), 3230-3237 (2002).

4. Luchsinger, J. A., Tang, M. X., Shea, S. \& Mayeux, R. Antioxidant vitamin intake and risk of Alzheimer disease. Arch Neurol. 60(2), 203-208 (2003).

5. Devore, E. E. et al. Dietary antioxidants and long-term risk of dementia. Arch Neurol. 67(7), 819-825 (2010).

6. Kato, Y. et al. Trends in dietary intakes of vitamins A, C and E among Japanese men and women from 1974 to 2001. Public Health Nutr. 12(9), 1343-1350 (2009).

7. Yamagishi, K. et al. The Circulatory Risk in Communities Study (CIRCS): A long-term epidemiological study for lifestyle-related disease among Japanese men and women living in communities. J. Epidemiol. 29(3), 83-91 (2019).

8. Ministry of Education, Culture, Sports, Science and Technology, Japan. Standard tables of food composition in Japan, 2015. (Official Gazette Co-operation of Japan, 2015). (in Japanese).

9. Tamiya, N. et al. Population ageing and wellbeing: Lessons from Japan's long-term care insurance policy. Lancet 378(9797), 1183-1192 (2011).

10. Ikeda, A. et al. Cigarette smoking and risk of disabling dementia in a Japanese rural community: A nested case-control study. Cerebrovasc. Dis. 25(4), 324-331 (2008).

11. Noda, H., Yamagishi, K., Ikeda, A., Asada, T. \& Iso, H. Identification of dementia using standard clinical assessments by primary care physicians in Japan. Geriatr. Gerontol. Int. 18(5), 738-744 (2018).

12. Iso, H., Rexrode, K., Hennekens, C. H. \& Manson, J. E. Application of computer tomography-oriented criteria for stroke subtype classification in a prospective study. Ann. Epidemiol. 10(2), 81-87 (2000).

13. Imano, H. et al. Non-fasting blood glucose and risk of incident coronary heart disease in middle-aged general population: The Circulatory Risk in Communities Study (CIRCS). Prev. Med. 55(6), 603-607 (2012).

14. Shimamoto, T. et al. Trends for coronary heart disease and stroke and their risk factors in Japan. Circulation 79(3), 503-515 (1989).

15. Imano, H. et al. Trends for blood pressure and its contribution to stroke incidence in the middle-aged Japanese population: The Circulatory Risk in Communities Study (CIRCS). Stroke 40(5), 1571-1577 (2009).

16. Uto-Kondo, H. et al. $\gamma$-Tocopherol accelerated sodium excretion in a dose-dependent manner in mats with a high sodium intake. J. Clin. Biochem. Nutr. 41(3), 211-217 (2007).

17. Gimenez-Llort, D. L. F. M., De la Fuente, M. \& Gimenez, L. Function and redox state of peritoneal leukocytes as preclinical and prodromic markers in a longitudinal study of triple-transgenic mice for Alzheimer's disease. J. Alzheimers Dis. 43(1), 213-226 (2015).

18. De la Fuente, M., Hernanz, A., Guayerbas, N., Victor, V. M. \& Arnalich, F. Vitamin E ingestion improves several immune functions in elderly men and women. Free Radic. Res. 42(3), 272-280 (2008).

19. Dong, Y. et al. Do low-serum vitamin E levels increase the risk of Alzheimer disease in older people? Evidence from a meta-analysis of case-control studies. Int. J. Geriatr. Psychiatry. 33(2), e257-e263 (2018).

20. Farrer, L. A. et al. Effects of age, sex, and ethnicity on the association between apolipoprotein $\mathrm{E}$ genotype and Alzheimer disease. A meta-analysis. APOE and Alzheimer Disease Meta Analysis Consortium. JAMA 278(16), 1349-1356 (1997).

21. Gray, S. L. et al. Antioxidant vitamin supplement use and risk of dementia or Alzheimer's disease in older adults. J. Am. Geriatr. Soc. 56(2), 291-295 (2008).

22. Masaki, K. H. et al. Association of vitamin E and C supplement use with cognitive function and dementia in elderly men. Neurology 54(6), 1265-1272 (2000)

23. Kryscio, R. J. et al. Association of antioxidant supplement use and dementia in the prevention of alzheimer's disease by vitamin $\mathrm{E}$ and selenium trial (PREADViSE). JAMA Neurol. 74(5), 567-573 (2017).

24. Miwa, K., Okinaga, S. \& Fujita, M. Low serum alpha-tocopherol concentrations in subjects with various coronary risk factors. Circ. J. 68(6), 542-546 (2004).

25. Yamagishi, K. et al. Serum $\alpha$-linolenic and other $\omega-3$ fatty acids, and risk of disabling dementia: Community-based nested casecontrol study. Clin. Nutr. 36(3), 793-797 (2017).

26. Gu, Y., Nieves, J. W., Stern, Y., Luchsinger, J. A. \& Scarmeas, N. Food combination and Alzheimer disease risk: A protective diet. Arch Neurol. 67(6), 699-706 (2010).

27. Kubota, Y. et al. Reproducibility of 24 -hour dietary recall for vitamin intakes by middle-aged Japanese men and women. J. Nutr. Health Aging. 14(3), 196-200 (2010).

\section{Acknowledgements}

The authors thank that health care staff of Ikawa, Minami-Takayasu, and Kyowa. We also appreciate Dr Bryan J. Mathis and Ms Flaminia Miyamasu of the Medical English Communications Center, University of Tsukuba, for language revision. The full list of CIRCS Investigators is presented in reference ${ }^{7}$. This study was supported by Health and Labour Science Research Grants for Dementia (Grant numbers: H21-Ninchisho-Wakate-007 and H24-Ninchisho-Wakate-003) from the Ministry of Health, Labour and Welfare, Japan; by JSPS Kakenhi Grant numbers 26253043, 17H04121, and 18K10097; by Japan FULLHAPP; and by the Osaka University International Joint Research Promotion Programme with University College London. 


\section{Author contributions}

S.A., K.Y., K.M., R.K. and H.I. substantially contributed to the conception or design of the work. All the authors contributed to the acquisition, analysis, or interpretation of data for the work. S.A. drafted the manuscript, and K.Y., K.M., R.K., A.I., M.U., C.R., Y.K., M.H.-T., Y.S., I.M., H.I., T.S., T.O., A.K., M.K., and H.I. revised the manuscript critically for important intellectual content.

\section{Competing interests}

The authors declare no competing interests.

\section{Additional information}

Supplementary Information The online version contains supplementary material available at https://doi.org/ 10.1038/s41598-021-95671-7.

Correspondence and requests for materials should be addressed to K.Y.

Reprints and permissions information is available at www.nature.com/reprints.

Publisher's note Springer Nature remains neutral with regard to jurisdictional claims in published maps and institutional affiliations.

(c) (i) Open Access This article is licensed under a Creative Commons Attribution 4.0 International License, which permits use, sharing, adaptation, distribution and reproduction in any medium or format, as long as you give appropriate credit to the original author(s) and the source, provide a link to the Creative Commons licence, and indicate if changes were made. The images or other third party material in this article are included in the article's Creative Commons licence, unless indicated otherwise in a credit line to the material. If material is not included in the article's Creative Commons licence and your intended use is not permitted by statutory regulation or exceeds the permitted use, you will need to obtain permission directly from the copyright holder. To view a copy of this licence, visit http://creativecommons.org/licenses/by/4.0/.

(C) The Author(s) 2021 